\title{
diverse: an R Package to Analyze Diversity in Complex Systems
}

\author{
by Miguel R. Guevara, Dominik Hartmann, and Marcelo Mendoza
}

\begin{abstract}
The package diverse provides an easy-to-use interface to calculate and visualize different aspects of diversity in complex systems. In recent years, an increasing number of research projects in social and interdisciplinary sciences, including fields like innovation studies, scientometrics, economics, and network science have emphasized the role of diversification and sophistication of socioeconomic systems. However, so far no dedicated package exists that covers the needs of these emerging fields and interdisciplinary teams. Most packages about diversity tend to be created according to the demands and terminology of particular areas of natural and biological sciences. The package diverse uses interdisciplinary concepts of diversity—like variety, disparity and balance- as well as ubiquity and revealed comparative advantages, that are relevant to many fields of science, but are in particular useful for interdisciplinary research on diversity in socioeconomic systems. The package diverse provides a toolkit for social scientists, interdisciplinary researcher, and beginners in ecology to (i) import data, (ii) calculate different data transformations and normalization like revealed comparative advantages, (iii) calculate different diversity measures, and (iv) connect diverse to other specialized R packages on similarity measures, data visualization techniques, and statistical significance tests. The comprehensiveness of the package, from matrix import and transformations options, over similarity and diversity measures, to data visualization methods, makes it a useful package to explore different dimensions of diversity in complex systems.
\end{abstract}

\section{Introduction}

While measuring diversity is a natural topic in ecology and biology, the rise of complexity and big data research has also provided new opportunities for researchers in various fields of social sciences to understand the evolution of diversity in social, economic, and political systems (Nature, 2014).

Today, a large range of scientific fields make use of diversity measures, including ecologists calculating the diversity of species (Humphries et al., 1994), sociologists measuring the structure of communities (Haughton and Mukerjee, 1995), economists studying the diversification of exports or financial assets (Hidalgo and Hausmann, 2009), scientometrists analyzing the diversity and interdisciplinarity of research fields (Rafols, 2014; Chavarro et al., 2014; Wagner et al., 2011), and computer scientists searching for new diversity methods to ensemble algorithms (Kuncheva and Whitaker, 2003).

Consequently, different fields of science have created several specialized R packages on diversity. This includes packages that allow for the analysis of species and biodiversity, (e.g. entropart (Marcon and Hérault, 2015), vegan (Oksanen et al., 2016), biodiversityR (Kindt and Coe, 2005)), social distances (e.g. Blaunet (Wang et al., 2016)), genetics (e.g. diveRsity (Keenan et al., 2013)), biological systems (e.g. divo (Pietrzak et al., 2016)), functional ecology (e.g. FD, (Laliberté and Legendre, 2010)), species complexity (e.g. hierDiversity (Marion et al., 2015)), bootstrapping diversity indices (e.g. simboot (Scherer and Pallmann, 2014)), disparity of phylogenetic trees (e.g. treescape (Jombart et al., 2016)) or phylogenetic patterns (e.g. SYNCSA (Debastiani and Pillar, 2012)).

Most packages on diversity are in the fields of ecology, biology and other natural sciences. Each discipline and respective package uses the particular terminology of its scientific field. It is important to translate the existing mathematical diversity formulas into the relevant concepts and language of each community, and thereby also helps to create new specialized measures considering the particular research topics and demands of each community. But the thematic specialization can also make interdisciplinary communication difficult and reduces the chances of the adoption of these new measures, concepts and specialized packages by researchers outside of the particular scientific field.

In recent years, an increasingly large number of research projects in social and interdisciplinary sciences are exploring the role of diversity in complex socioeconomic systems. These new approaches in social and interdisciplinary sciences use existing diversity concepts from biological and natural sciences, but also have their own particular needs and concepts. For instance, recent work in economics, scientometrics and network science has highlighted the importance of diversification processes in complex systems, such as research, financial and energy portfolios, cultural diversity, the diversity of ties in social and economic networks, or the emergence of new or related scientific and economic fields (Hidalgo et al., 2007; Frenken et al., 2007; Rafols et al., 2010; Chavarro et al., 2014; Guevara et al., 2016; Eagle et al., 2010; Farchy and Ranaivoson, 2011).

Here we present the package diverse which aims to provide a useful toolkit for social scientists and interdisciplinary teams to measure and visualize diversity in socioeconomic systems, by providing 
several of the most used measures of diversity and allowing for versatility with existing $R$ packages on diversity, focusing, for example, on the calculation of similarity and distance measures (proxy (Meyer and Buchta, 2015)); bias corrected diversity measures (entropart (Marcon and Hérault, 2015)); or the visualization of diversity in matrices, treemaps and networks (pheatmap (Kolde, 2015), treemap (Kindt and Coe, 2005), and igraph (Kindt and Coe, 2005)).

The package applies a diversity taxonomy that includes the variety, balance and disparity of complex systems (Stirling, 2007). The package diverse allows researchers to:

1. Read input and process data from complex systems in a simple manner.

2. Compute some of the most commonly used measures of diversity across sciences-including Shannon-Entropy, Herfindahl-Hirschman Index, Gini-Simpson Index or Berger-Parker Index.

3. Calculate complementary measures that are related to diversity, such as ubiquity, disparity or similarity between categories and entities.

4. Apply advanced diversity measures such as Rao-Stirling diversity and other diversity measures including weighting parameters.

5. Visualize different dimensions of diversity as variety, balance, or disparity.

The package diverse is available within CRAN. The newest development version is accessible at the branch development of the Git repository github.com/mguevara/diverse. In this Git repository interested users are also very welcome to submit issues.

The remainder of the article is organized as follows. In Section Diversity we describe different dimensions of diversity. In Section Input data we explain which type of data can be read/imported into the package and how it can be normalized, using for instance either binary, absolute or relative values. In Section Measuring diversity we present the measures available in this package discussing how researchers can use them to calculate different dimensions of diversity. In Section Synthetic data and performance tests we explain functions that are included in the package to simulate data and conduct bias, coverage, and performance tests. In Section Conclusions we summarize and briefly discuss the limitations and advantages of the package.

\section{Diversity}

In this section we explain key properties of diversity with the help of example datasets.

\section{Example data}

To illustrate the use of the package diverse, we will work with three datasets: Pantheon, Scidat and Geese.

- Pantheon is a sample of 10 countries from MIT's Pantheon project (Macro Connections MIT MediaLab, 2014). This dataset allows for a comparison of the diversity of occupations of the globally famous people from each country. The complete dataset includes 11341 persons classified in 88 distinct occupations and assigned to 195 countries (Yu et al., 2016).

- Scidat is an aggregation of the number of scientific publications assigned to 27 areas of science. This dataset was aggregated over the raw data of SCImago (2007). Scidat includes a sample of 10 countries from the year 2013.

- The third dataset is on the geese population in the Netherlands and was published by the Sovon Dutch Centre for Field Ornithology (Nederland, 2015). This dataset presents observations of 4 species of geese over a period of 11 years.

The three datasets are included in the package diverse. The subset of the Pantheon dataset is included as a "data. frame" object and both the Scidat and Geese datasets are included as a "matrix" object.

\section{The actors and concepts of diversity}

We use the term entity to describe the systems or agents that host a set of categories. Entities could be, for example, persons, companies, countries, regions, institutions, or years.

We also use the term category to identify the different types of species that define the diversity of an entity. Categories could include types of animals, species of plants, fields of research, taxonomies of products, or technologies. The package assumes that the imported dataset has a previously given classification scheme. 
The terms value or value of abundance is used for the amount of a category in each entity. This could be the quantity of each species in an ecosystem, or the total value of the different types of export goods of an economy.

In Pantheon, entities are countries, categories are different types of occupations, and values are the respective number of globally famous persons a country has in each category. In Scidat, entities are countries, categories are SCimago's areas of science, and values are the total number of citable documents that a country has published in each area in 2013. In Geese, entities are years, categories are species of geese, and values are the number of each species of geese observed in the Netherlands in the respective year.

Pantheon is a good example of data where some entities have missing values in some categories. Scidat is a useful example where most entities have values in each category yet have very large absolute differences between their values. The Geese dataset is a good example of the temporal evolution of natural species.

It must be noted that in most diversity measures (e.g. variety or Shannon entropy) the information about the number and types of categories of a single entity is sufficient to calculate this entity's diversity. However diverse is oriented to also work with multiple entities. Therefore it allows for the calculation of different distance and similarity matrices across categories and entities, and uses these distance measures in diversity measures like the Rao-Stirling Index (Stirling, 2007). Moreover, diverse allows for the calculation of relative specialization measures like the activity index or revealed comparative advantages (RCAs) that takes the portfolio and size of other entities into account when evaluating their relative specialization or comparative advantages (e.g. Belgium versus USA) (see Section Data transformation and normalization). Subsequently, we will mainly use data examples with multiple entities and categories. Nonetheless, many measures embedded in diverse can also be used to track the evolution of the diversity within a single entity.

Regarding the concept of diversity, previous interdisciplinary studies on diversity (Rao, 1982; Stirling, 1998; Mcdonald and Dimmick, 2003; Stirling, 2007) showed that the concept of diversity is related to three main questions:

1. How many categories does an entity (and/or does each entity in a system) have?

2. How much of each category does an entity (and/or each entity in a system) have?

3. How distinct are the categories of an entity (and/or the categories of each entity in a system)?

Stirling $(1998,2007)$ categorized these three properties of diversity as variety, balance, and disparity. Most diversity measures combine and emphasize these aspects with varying weights. Comprehensive measures take all three dimensions deliberately into account. Moreover data visualization methods and R packages like, for instance, treemap (Tennekes, 2016) or igraph (Csardi and Nepusz, 2006) can help to visualize these three dimensions of diversity. For instance, treemaps-allow for an emphasis on variety and balance (Hausmann et al., 2011, p.105)—or network overlays maps allow for an emphasis on disparity (Hidalgo et al., 2007; Rafols et al., 2010). The disparity dimension is often implied by a previous classification scheme, like a given classification of types of animals, scientific fields or exports, phylogenetic trees and/or it can alternatively be calculated based on a similarity or distance matrix (see also Section Matrix of dissimilarities between entities).

As an example, Figure 1 presents treemaps about the diversity of occupations of globally famous individuals from Canada and Uruguay according to MIT's Pantheon. Variety is represented by the number of boxes, balance is indicated by the differences in the size of the boxes (= percentage of the category), and disparity is represented by different colors.

First, regarding the variety, it is clear that Canada has a larger number of different occupations ( 27 boxes in Figure 1a) than Uruguay ( 4 boxes in Figure 1b). Second, regarding the balance, we can observe that Uruguay's concentration in terms of soccer players is very high (52.63\%), while Canada's balance is less concentrated on one category, but is spread across more occupations of the Pantheon dataset. The $\mathrm{R}$ package treemap or other specialized data visualization programs like D3plus allows for the creation of such treemaps with different colors, text sizes and further visualization options.

To illustrate disparity, Figure 2a shows a similarity network between areas of science that was obtained by considering each column as a vector of features (i.e. the number of articles of each country in that category) and then computing the cosine (dis-)similarity between those vectors. The pre-process options embedded in the package allows for the calculation of different types of similarity and distance matrices and considers both the absolute shares as well as relative strengths/specializations/comparative advantages of entities (see Section on Data transformation and normalization).

As expected, we can observe that "Social Sciences" are close to "Arts and Humanities," but more distant from "Mathematics" or "Engineering." We can also see the clustering between natural sciences and technological fields, like "Chemistry" and "Material Sciences." The disparity within a country is high if the dissimilarities between the areas/categories, in which the country has values, are also high. 


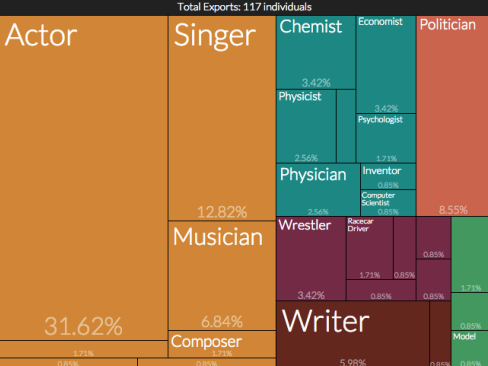

(a) Canada

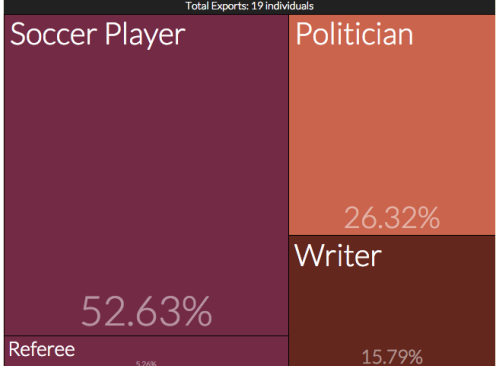

(b) Uruguay

Figure 1: Globally famous people according to Pantheon dataset. The size of the boxes is proportional to the number of people assigned to each occupation and born in that country. The color is according to main domains of occupations. Source http://pantheon. media.mit. edu.

In so-called overlay networks (Rafols et al., 2010), the values of entities (which are countries in the present dataset) are overlaid on the global network structure. Moreover, the variety is represented by the number of colored nodes, and the dimension balance can be represented by the size of the nodes. Figure 2b illustrates that in the Scidat example, Germany has comparative advantages (see the section on Data transformation and normalization for details) in many fields of science across the network, while China (Figure 2c) is more specialized (concentrated) in technological areas and engineering. In consequence the disparity, variety and balance in Germany are higher than in China. Such network overlay maps can, for instance, be made with the R package igraph.

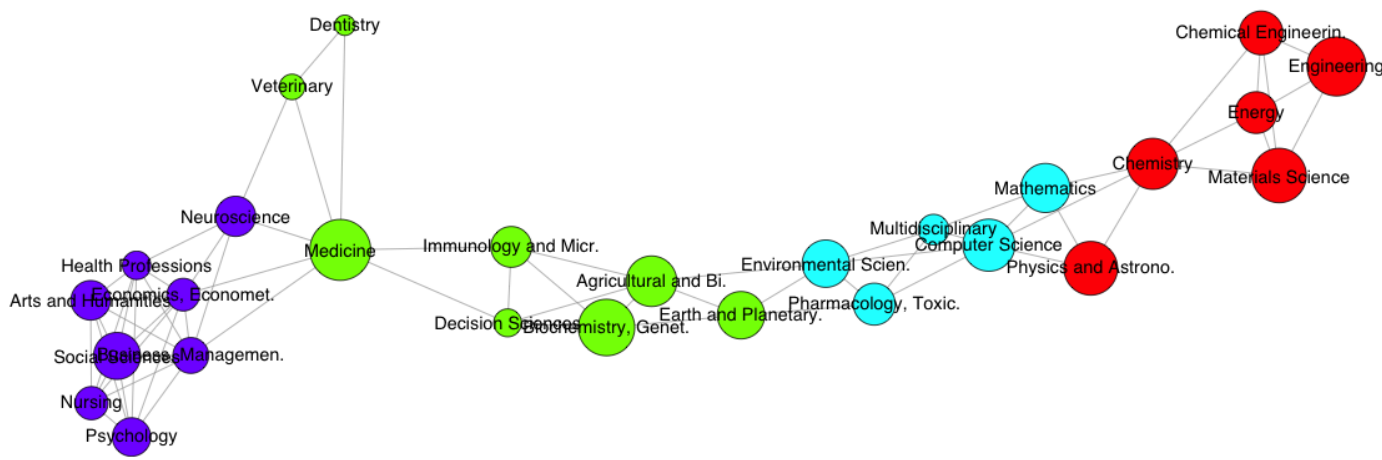

(a) Network of similarities between areas of science.

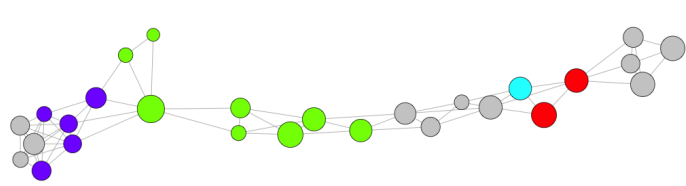

(b) Overlay network for Germany.

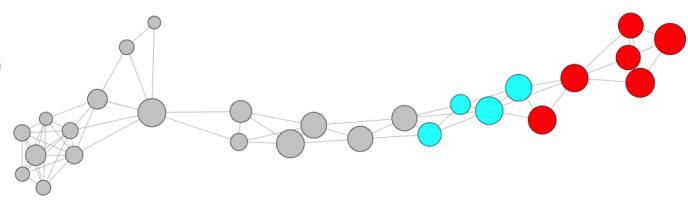

(c) Overlay network for China.

Figure 2: Cosine similarity network of 27 areas of science obtained with Scidat dataset. Links represent the (dis)similarities between areas. Links below the threshold of 0.015 of Cosine similarity are not illustrated. The force-directed algorithm Fruchterman-Reingold was used for the network layout. The size of the nodes in 2a represents the total number of papers authored by the 10 countries included in Scidat; the size of the nodes in $2 \mathrm{~b}$ and $2 \mathrm{c}$ is proportional to the papers authored for each country. Colors are according to communities detected by the algorithm fastgreedy. The grey-colored nodes identify areas with Revealed Comparative Advantages RCA below 1 (see section on Data transformation and normalization for details).

In the following sections we will detail how to import and transform data, and how to use the diverse package to quantify the described properties of diversity. 


\section{Input data}

This section details the type of the data object that is required by the package, how to import data from an external data file, and how to pre-process or normalize the raw data.

\section{Input formats}

Since diverse was created to be able to work with multiple categories $(N)$ and multiple entities $(M)$ simultaneously, the data objects used for most of the functions in the package diverse can be either a data frame or a matrix of size $M \times N$.

In the case of a data frame-meaning that the data is shaped as an edge list-it has to have three columns in this order: entity, category and value. The first two columns are of the type "factor" and the third column is of type "numeric". The pantheon data frame is an example of this type of data object.

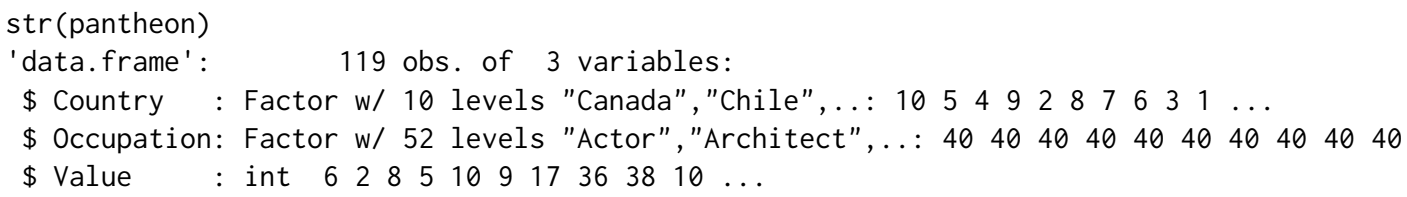

When the data is in a "matrix" format, each cell has to contain numeric values and the rownames and colnames must be defined with the names of entities and the names of categories. Non-existent values (NA) or 0 have to be used to indicate the lack of a category in an entity. The matrix of the scidat dataset is an example of this type of object.

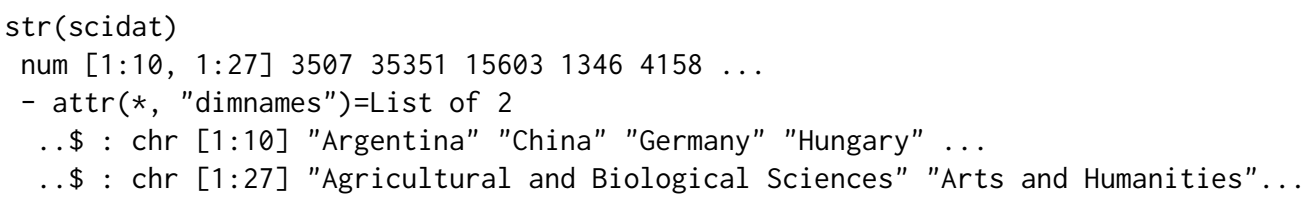

If the matrix has categories in the rows and entities in the columns, the parameter category_row must be set to TRUE when using the functions included in diverse. The matrix of the geese dataset is an example of this kind of object.

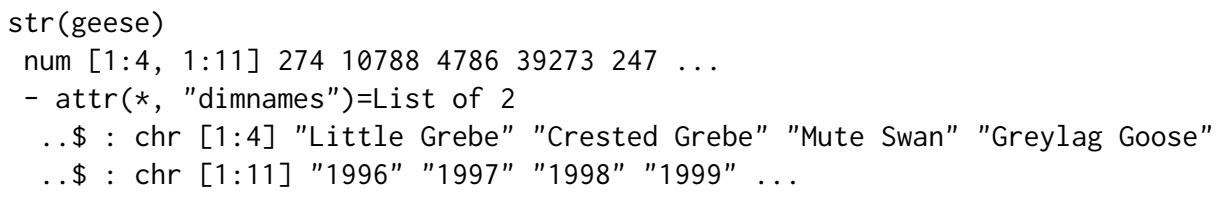

\section{Importing data}

To simplify the input of data from external files, diverse includes the function read_data(). This function reads CSV files and automatically detects whether the data file is a matrix or an edge shape. Moreover, it retrieves a data frame ready to be passed to the parameter data of all the functions included in diverse. The user has to provide the path to the external CSV file by using the argument path.

In addition, to facilitate the import of data from different software formats, the function read_data() includes the parameter type that can be used to indicate whether the external data file comes for instance from Stata or SPSS. This functionality depends on the package foreign (R Core Team, 2015).

\section{Data transformation and normalization}

Depending on the characteristics of the dataset, researchers often need to normalize, transform or filter the data before measuring diversity. For instance, in some cases the absolute quantity or share of each category in the portfolio of an entity is important. In other cases, the relative specialization and diversification of entities in comparison to a set of other entities (e.g. revealed comparative advantages of countries, or the relative activity in certain research fields) is more important. There are also cases where binary values (e.g. are certain categories present or not present) or discrete steps (e.g. not present, low, middle, high value) are important, depending on the respective research question. For this purpose, diverse includes the function values() which allows for the filtering and exclusion of data below a certain threshold value, and to binarize or normalize the data. 
The normalization process could include forming proportion values, Revealed Comparative Advantages (RCA) (Balassa, 1986) and normalized RCAs (which is also called Activity Index). With the term proportions we refer to normalization within an entity (dividing the value of an entity in each category by the sum of values of the entity in all categories). The calculation of RCAs or the Activity Index is a normalization related to the other entities. For instance in economics, RCA computes the ratio between the proportion of a category within an entity, e.g. a country or region, and the proportion that represents that category in the global system (e.g. the world economy). The purpose of this measure is to understand in which categories an entity is relatively more specialized than others and thus seems to have a comparative advantage (Balassa, 1965). Typically, values of RCA greater than 1 are considered to "reveal" comparative advantages in the respective categories. Values below 1 reveal comparative disadvantages. The same idea can be found in Scientometrics where the RCAs are normalized between -1 and 1 , and named "Activity Index." Both options, RCA and Activity Index are included in diverse.

To use these functionalities, the arguments norm, filter, and binary should be used. Argument norm can be set, for instance, to ' $p$ ' for proportions, ' $r c a$ ' for RCAs, or 'ai' for Activity Index. The argument filter allows the user to indicate a threshold, below which all the values are discarded (replaced with NA). The argument binary has to be set to TRUE if binary values are required. If the three arguments are applied, then the function values() first applies the normalization, then the filter and finally creates binary values.

The following matrix visualizations show the importance of the normalization process in datasets like Scidat where most entities produce all categories and the absolute differences (e.g. between the values of a small and a large country) are very large.

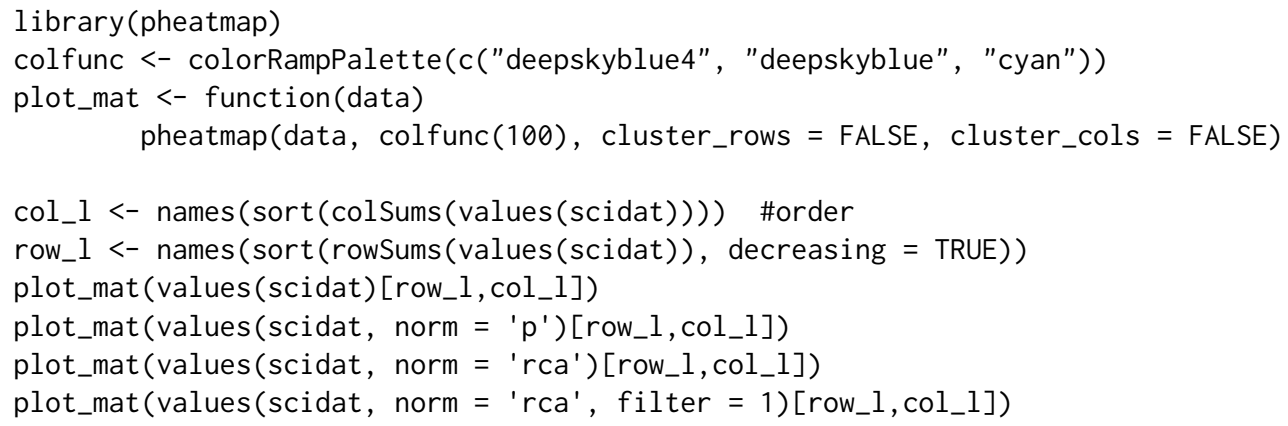

In Figure $3 a$ we see the absolute values of authored papers by country in each area. The large number of papers from the United States in "Medicine" and "Biochemistry", as well as from China in "Engineering" and "Material Sciences" are the outstanding features of this matrix. If we consider proportions instead of absolute values, we can observe that "Medicine" is an important field of science for most countries, while a large proportion of the publication portfolios in Argentina or Mexico are in agricultural and biological sciences (see Figure 3b). Moreover, if we want to compare the relative specialization and comparative advantages of each country within the global system, an RCA based matrix will be more useful. Figure 3 c presents the values of all RCAs, while Figure $3 \mathrm{~d}$ presents the values of an RCA matrix in which values below 1 are represented by empty cells.

\section{Measuring diversity}

In this section we explain the measures included in the package diverse by illustrating their use with our sample datasets.

\section{Measures included}

The diversity measures included in the package diverse allow for different dimensions of diversitylike variety, balance and disparity—-to be analyzed separately or jointly.

To compute these measures, the main function diversity() must be used. All diversity measures available in the package diverse are listed in Table 1. These measures are organized from simple to complex, considering the properties of diversity they take into account.

Regarding the parameters of the function diversity(), the dataset to be analyzed should be provided in the data parameter, and the required diversity measure(s) should be provided in the type parameter.

The argument data has to fulfill the characteristics analyzed in the previous section on Input data. The argument type can be a single string or a vector of strings, with either the complete name of the 

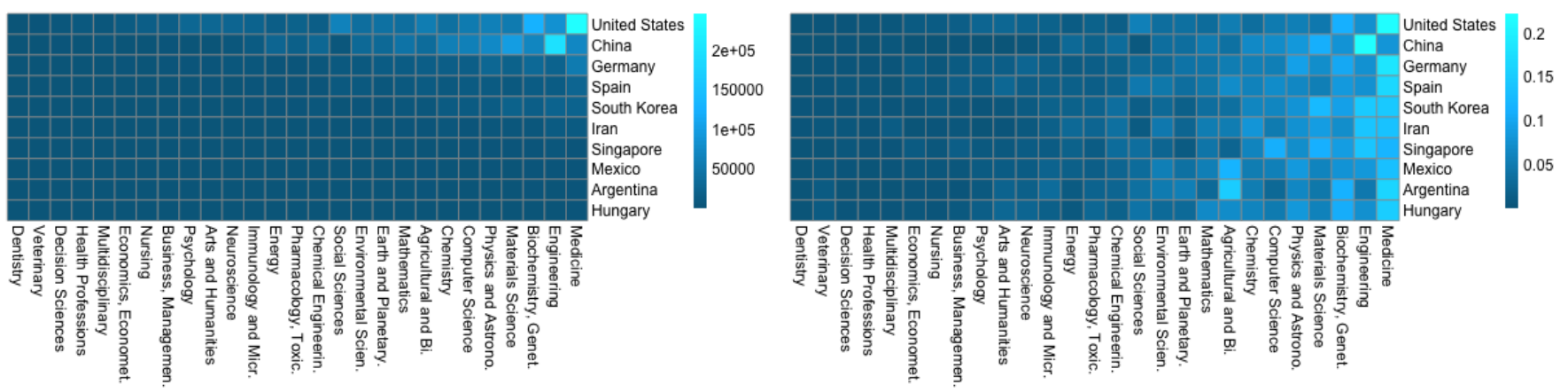

(a) Raw values

(b) Proportions
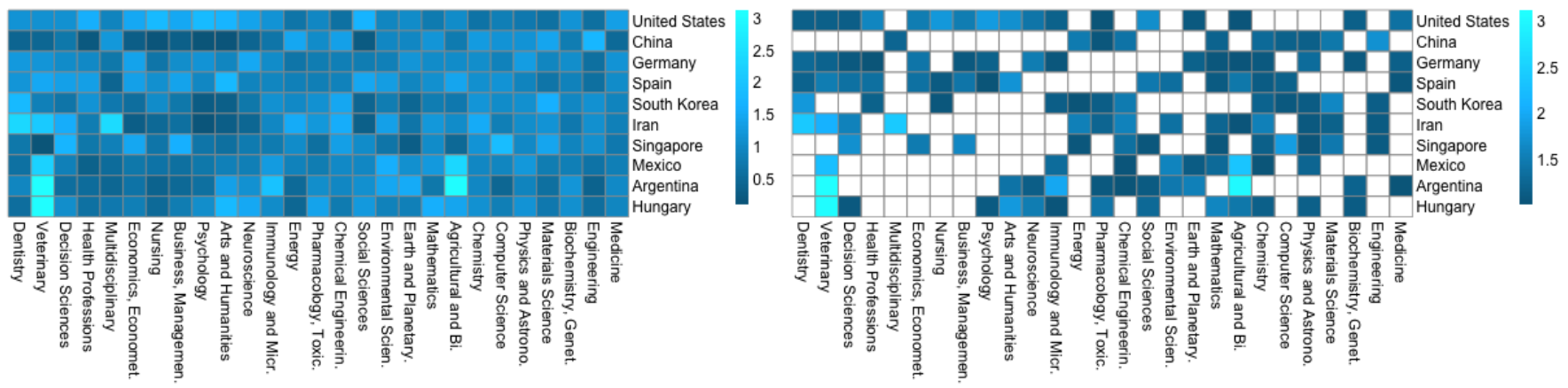

(c) RCA

(d) RCA filtered

Figure 3: Pheatmaps for matrices. The lighter the color, the higher the value. The white color represents empty cells.

\begin{tabular}{clll} 
ID & Measure & Formula & Reference \\
\hline $\mathrm{v}$ & Variety & $v=\sum_{i}\left(p_{i}^{0}\right)$ & \\
hhi & Herfindahl-Hirschman Index & $H H I=\sum_{i}\left(p_{i}^{2}\right)$ & Rhoades (1993) \\
$\mathrm{b}, \mathrm{gs}$ & Blau Index, Gini-Simpson & $B=1-\sum_{i}\left(p_{i}^{2}\right)=1-H H I$ & Blau (1977); Gini (1912) \\
$\mathrm{s}$ & Simpson & $D_{S}=\sum_{i} n_{i}\left(n_{i}-1\right) / N_{t}\left(N_{t}-1\right)$ & Simpson (1949) \\
$\mathrm{bp}$ & Berger-Parker & $D_{B P}=\max _{i}\left(p_{i}\right)$ & Berger and Parker (1970) \\
$\mathrm{e}$ & Shannon Entropy & $H=-\sum_{i}\left(p_{i} \log p_{i}\right)$ & Shannon (1948) \\
ev & Pielou Evenness & $J=-\sum_{i}\left(p_{i} \log p_{i}\right) / \log v$ & Pielou (1970) \\
re & Rényi-Entropy & ${ }^{q} H=(1-q)^{-1} \log \left(\sum_{i} p_{i}^{q}\right)$ & Rényi (1961) \\
hcdt & HCDT Entropy & ${ }^{q} H=(q-1)^{-1}\left(1-\sum_{i} p_{i}^{q}\right)$ & Havrda and Charvát (1967); Tsallis (1988) \\
hn & Hill Numbers & ${ }^{q} D_{H N}=\left(\sum_{i} p_{i}^{q}\right)^{1 /(1-q)}$ & Hill (1973) \\
\hline $\mathrm{d}$ & Disparity & $D I S=\sum_{i j} d_{i j} / N$ & \\
rao & Rao & $D_{R A O}=\sum_{i j} d_{i j} p_{i} p_{j}$ & Rao (1982) \\
rs & Rao-Stirling & $\Delta=\sum_{i j} d_{i j}{ }^{\alpha}\left(p_{i} p_{j}\right)^{\beta}$ & Stirling (2007) \\
\hline & & &
\end{tabular}

Table 1: Summary of measures available in the package diverse. The first block of measures are associated mainly with the dimensions variety and balance of the diversity, while the second block presents measures that use also the dimension disparity. $C$ is the set of categories present in the entity. $i, j \in C . i \neq j$ and $i j \neq j i ; n_{i}$ is the value of abundance and $p_{i}$ the proportion of the category $i$ in the entity. $v=n(C)$ is the number of categories present in the entity-the variety. $N_{t}=\sum n_{i}$. Here $\log$ is the logarithm usually natural, and $q, \alpha, \beta \geqslant 0$. For HCDT and Rény entropies when $q \rightarrow 1$ converge to the Shannon entropy. Additionally, for Hill numbers, when $q \rightarrow 1$, it results in the exponential of Shannon Entropy. 
measure or a mnemonic term (see column ID in Table 1). In the following sections we will explain the function diversity() and the related functions variety(), balance(), and disparity().

\section{Variety or richness}

Variety measures how many categories or types an entity has. Variety is useful as a first approach to the diversity of an entity since the number of categories (e.g. species, scientific fields or export categories) is easy to understand and calculate. Users can compute variety both within the function diversity() indicating type $=$ ' $v$ ', or with the function variety () . Both options return a data frame with the values of variety. In the case of the function variety() values are sorted in an decreasing order. For an increasing order, the argument decreasing should be set to FALSE.

For instance, we can compare the variety of the 10 countries included in our sample of Pantheon. Canada and China rank at the top of variety, while Uruguay and Vietnam rank at the bottom. In Scidata, US and Germany (see Figure $2 b$ ) have the highest level of variety, while China (see Figure 2c) and Mexico have the lowest variety. It is important to note that we are only considering fields of science in which these countries have Revealed Comparative Advantages (RCAs) equal to or higher than 1 .

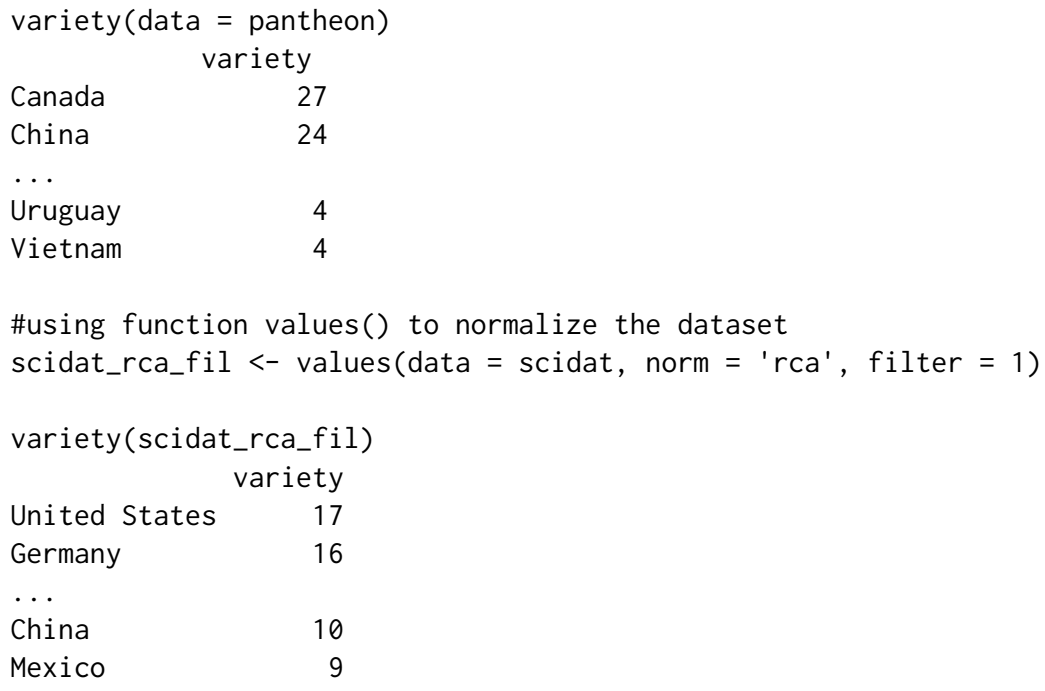

Being related to the concept of variety, it is helpful in some cases to know the ubiquity or rareness of each category by considering its presence in all entities. Ubiquity could also be considered as the variety of entities that each category has (Hidalgo et al., 2007). We include this concept and measure through the function ubiquity () that returns the number of entities in which the category is present. A decreasing order is retrieved by default. In our sample of Pantheon "politicians" and "soccer players" are more common (ubiquitous) than "referees" or "wrestlers."

ubiquity (data = pantheon)

$\begin{array}{lr} & \text { ubiquity } \\ \text { Politician } & 10 \\ \text { Writer } & 8 \\ \text { Soccer.Player } & 6 \\ \ldots . & \\ \text { Referee } & 1 \\ \text { Wrestler } & 1\end{array}$

\section{Diversity measures that emphasize abundance and balance}

Balance measures how much of each category the entity has. The raw indicators of balance are the values of abundance or the relative values of abundance which are the proportions $p_{i}$ of each $i$-th category. The package diverse includes the function balance() which retrieves the matrix of entities-categories with their correspondent shares, proportions or probabilities.

The word balance is used when the values of abundance are more equally distributed across the categories. For a given variety, a more balanced system is considered more diverse. Extreme cases are those where the quantity of elements for each category is exactly the same (i.e. perfect balance) or conversely, where all the elements are concentrated in just one category (i.e. total concentration). 
As pointed out by Tuomisto (2012), measuring balance alone is a complicated task because it is difficult to remove the effect of variety on it. The only measure of "balance" in a strict sense that is facilitated in this package is Pielou's evenness (Pielou, 1970), which according to Jost (2010) is also the best measure of balance.

However, diverse also allows for the calculation of a series of commonly used "balance" measures like the Herfindahl-Hirschman Index (HHI), Gini-Simpson or Blau-Index that emphasize the evenness or balance of a system (while also being affected by the variety of categories).

Diversity measures related to the property "balance" could be understood as statistical dispersion and are mainly a function of $p_{i}$. While some of them measure the evenness or heterogeneity of the distribution such as the Blau Index, others emphasize the concentration, such as the HerfindahlHirschman Index (HHI).

The Herfindahl-Hirschman Index (HHI), for example, computes the probability that two individuals taken randomly belong to the same category. This probability is calculated with replacement, which means that after taking the first individual into account, it is replaced with an identical one; and thus neither affecting the total number of individuals in that category $\left(n_{i}\right)$ nor the total amount of individuals in the entity $\left(N_{t}\right)$. HHI is used in economics, for instance, to estimate the concentration of markets or wealth (Ceriani and Verme, 2011).

Taking into account that balance is the opposite to concentration, the Gini-Simpson Index $(1-H H I)$ subtracts $H H I$ from 1 to estimate balance. The same idea is behind the Blau Index. The Blau Index was created to measure the heterogeneity of social communities and its use is very common in sociology and other social sciences.

Similar to HHI, Simpson measure $D_{S}$ has the same probabilistic idea of measuring concentration, but it computes the probability without replacement-meaning that the values of $N_{t}$ and $n_{i}$ decreases in 1 after the first probability is calculated (see Table 1). This measure of concentration and its equivalent balance or index of diversity $\left(1-D_{S}\right)$ are widespread in ecology. Moreover, the reciprocal index $\left(R_{S}=1 / D_{S}\right)$ can be calculated.

In the following example, the Herfindahl-Hirschman Index (HHI), the Gini-Simpson Index and the Blau Index from Pantheon are computed by using the function diversity(). We can observe that Uruguay and Vietnam have a higher HHI value and are thus more concentrated and less balanced than Canada and Chile. Note that the opposite occurs with the Gini-Simpson or Blau indexes. Besides the Gini-Simpson index, the concentration gini.simpson. C and the reciprocal of the concentration gini.simpson. $R$ are also retrieved.

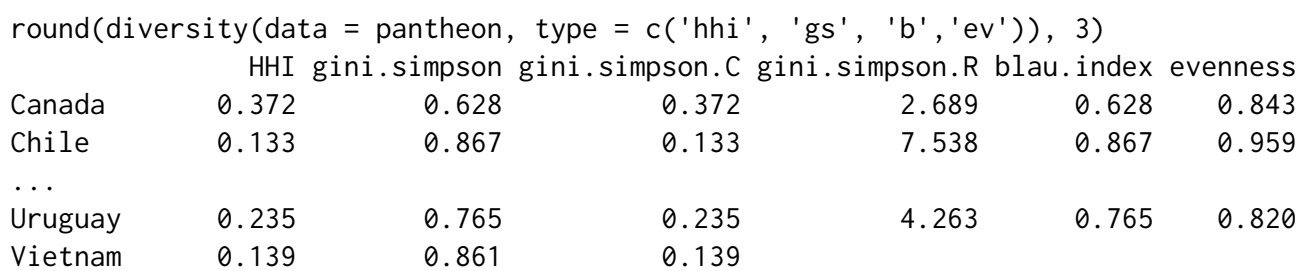

Graphical representations can help to understand the importance of balance and how it is captured by specific diversity measures. Figure 4 a illustrates how the share of the dominant species Greylag Goose increases over time and how the share of Crested Grebe declines. The result is an unbalance between the species in this ecosystem.

The decrease in diversity of geese- understood here mainly as the balance of the abundance of different types of geese-can, for instance, be captured by Berger-Parker measures. The BergerParker Dominance Index $\left(D_{B P}\right)$ is a measure based on the dominant category $\left(\max \left(p_{i}\right)\right)$ and thus captures the dominance of the Greylag Goose. On the other hand, the Berger-Parker Index of Diversity $\left(I_{B P}=1 / D_{B P}\right)$ captures the balance between the species. Figure $4 \mathrm{~b}$ shows how the Berger-Parker Index of Diversity decreases over time.

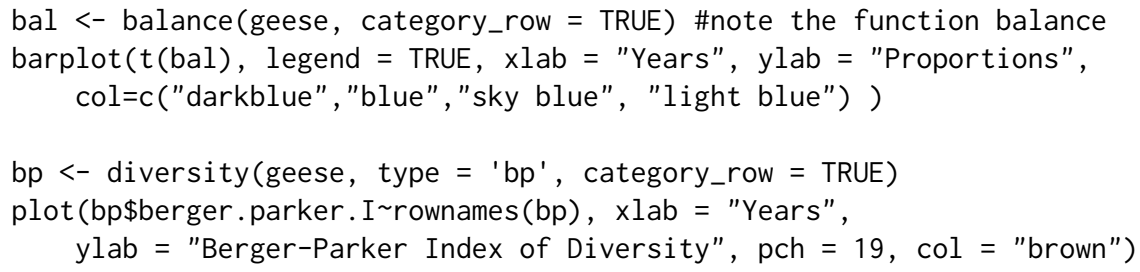




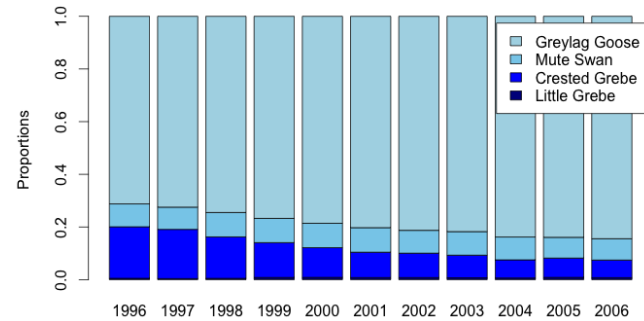

Years

(a) Proportions of geese species.

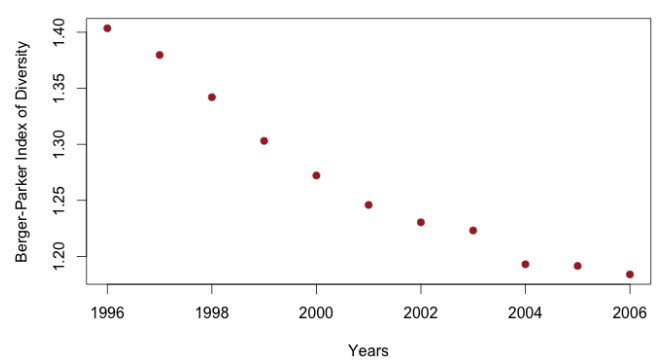

(b) Berger-Parker Index of diversity.

Figure 4: Analysis of balance for Geese dataset.

$\begin{array}{lrrrrrrr}\text { entropy } & \text { evenness } & \text { simpson.D simpson. I } & \text { simpson. } & \text { berger.parker.D } & \text { berger.parker. I } \\ 1996 & 0.7993160 & 0.5765846 & 0.5534977 & 0.4465023 & 1.806692 & 0.7124871 & 1.403534 \\ 1997 & 0.7764028 & 0.5600563 & 0.5674638 & 0.4325362 & 1.762227 & 0.7247953 & 1.379700 \\ \ldots & & & & & & & \\ 2005 & 0.5790954 & 0.4177290 & 0.7160910 & 0.2839090 & 1.396471 & 0.8392823 & 1.191494 \\ 2006 & 0.5633026 & 0.4063369 & 0.7245616 & 0.2754384 & 1.380145 & 0.8446653 & 1.183901\end{array}$

\section{Entropy measures and Hill numbers}

Shannon Entropy is a frequently used measure of balance and diversity. Entropy is a measure first created and used in information theory (Shannon, 1948) and has been widely adopted by other disciplines such as computation, ecology, and economy.

Entropy $H$ measures the minimum volume of communication required to code a message. Furthermore, as pointed by Hidalgo (2015, p.17), "entropy is a measure of the multiplicity of states". A high value of multiplicity of states (categories) implies more evenness and less concentration: as a consequence, the higher the variety and the balance, the higher the entropy. In the example above we could observe how the entropy in Geese decreased from 0.78 in 1996 to 0.56 in 2006. This is a consequence of an increase of the population of the dominant species.

A generalization of Shannon Entropy that is also included in diverse is Rényi's entropy (see Table 1). Rényi's entropy allows the users to give more or less relative importance to rare categories through the parameter $q$.

Another parameterized entropy is the HCDT entropy (Havrda and Charvát, 1967; Daróczy, 1970; Tsallis, 1988). It is noteworthy that Variety, Shannon, Blau's and Berger-Parker's indexes are special cases of HCDT (respectively with the parameter $q=0,1,2$ and infinity).

Finally, Hill numbers (Hill, 1973) are a mathematically unified family of diversity indexes that differ only by a parameter $\mathrm{q}$ and that take the effective number of categories into account, i.e. the number of equally abundant species that would be needed to give the same value of a diversity measure (Chao et al., 2014b). Hill numbers are of particular interest since entropy is not linear to the number of categories hosted by an entity Jost (2006). Moreover, several widely used diversity indexes, like variety/richness, Shannon entropy, Gini-Simpson Index, Rényi's or HDCT entropy, can be obtained from Hill numbers (Chao et al., 2014a).

By using diverse we can observe the similarities between entropy measures and Hill numbers, when $q$ has values of 0,1 and 2 .

When $q=0$, variety, HCDT entropy and Hill numbers are the same. Rényi entropy is equal to $\log$ (variety). When $q=1$, Rényi entropy and HCDT entropy are equal to Shannon entropy $(H)$, while Hill numbers are equal to the exponential of $H$. When $q=2$, HCDT entropy is equal to Gini-Simpson, while the Hill numbers index is equal to the reciprocal (gini . simpson. R) of the index of concentration of Gini (or Herfindahl-Hirschman Index (gini. simpson. C)).

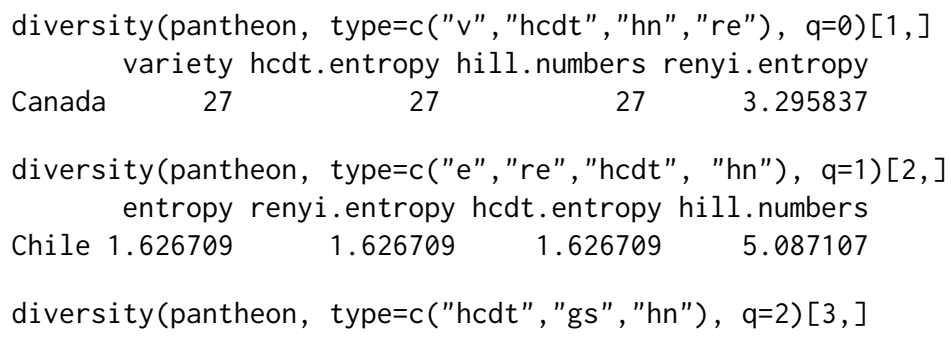




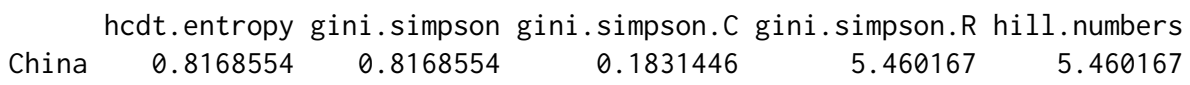

Generally for the three parameterized measures that are included in diverse (i.e. Rényi Entropy, HCDT and Hill numbers), the parameter value $q=0$ calculates variety, $q<1$ considers rare categories more important for diversity, $q=1$ considers all categories as equally important, and $q>1$ mainly shows the impact of dominant categories in diversity.

\section{Disparity}

Another significant dimension of diversity is the disparity or dissimilarity between categories or entities (Stirling, 2007; Rafols and Meyer, 2009). Disparity is important for all diversity measures, though, often pre-given in form of classification schemes, like, for example, phylogenetic trees or types of species in ecology, or the type of research fields in scientometrics. Here, we explicitly take the diversity dimension disparity into account.

The dimension of disparity provides a notion of how different the categories of an entity are. For example the areas "Mathematics" and "Physics" are arguably more similar than "Mathematics" and "Nursing." Measures of diversity, therefore, are also closely related to distance and similarity measures like Euclidean distances, cosine similarity, Jaccard-Index, or expert classifications of different categories.

\section{Matrix of dissimilarities between entities}

Beside computing disparity, the dissimilarity matrix between entities is also useful for the visualization of networks, such as those proposed to evaluate economic complexity (Hidalgo et al., 2007; Hartmann et al., 2016) or the research capabilities of scholars (Guevara et al., 2016). Moreover, it helps to analyze the portfolio of entities in so-called network overlay maps and to explore the path of diversification as a function of the disparity in the network (Rafols et al., 2010; Guevara et al., 2016).

Based on the 10 countries included in Scidat, we calculate the dissimilarities between categories and then we create a network of areas of science in the following example. The resulting network is the one presented in Figure 2a in Section Diversity.

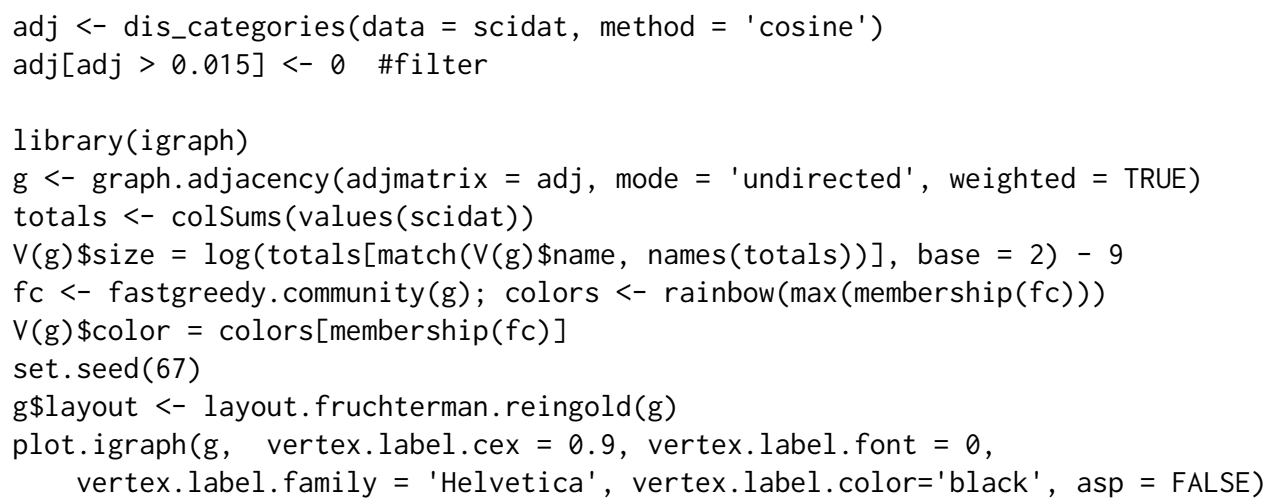

\section{Calculating dissimilarities between entities}

The function dis_entities() can be used to calculate a matrix of dissimilarities between entities. The following example computes the matrices of dissimilarities between countries (entities) for the 10 countries included in Scidat. In this example, Argentina is more similar to Mexico (0.04) and less similar to China (0.32). In addition, Germany is more similar to Hungary (0.02) and less similar to Singapore (0.10).

\begin{tabular}{|c|c|c|c|c|c|c|c|}
\hline & Argentina & China & Germany & Hungary & Iran & Mexico & Singapore... \\
\hline Argentina & 0.00 & 0.32 & 0.09 & 0.07 & 0.17 & 0.04 & 0.25 \\
\hline China & 0.32 & 0.00 & 0.20 & 0.19 & 0.06 & 0.18 & 0.06 \\
\hline Germany & 0.09 & 0.20 & 0.00 & 0.02 & 0.07 & 0.05 & 0.10 \\
\hline Hungary & 0.07 & 0.19 & 0.02 & 0.00 & 0.07 & 0.03 & 0.11 \\
\hline Iran & 0.17 & 0.06 & 0.07 & 0.07 & 0.00 & 0.07 & 0.05 \\
\hline Mexico & 0.04 & 0.18 & 0.05 & 0.03 & 0.07 & 0.00 & 0.13 \\
\hline
\end{tabular}




\section{Average or Sum Disparity}

The function disparity() computes the average and/or the sum of dissimilarities among categories, either based on a given dissimilarity matrix of the user or through calculating the dissimilarity matrix within the function. The first case is based on a matrix of dissimilarities that the user provides in the argument dis. The dissimilarity matrix has to include the same names of the categories in the rownames and in the colnames.

In the second case, when the argument dis is not provided, diverse computes the disparities by using the dissimilarity matrix calculated by using the previously detailed function dis_categories(), as in the following example where the argument dis is not defined.

In this example with Scidat, we note that the average dissimilarities of categories in the US are greater than the disparities in Argentina or China.

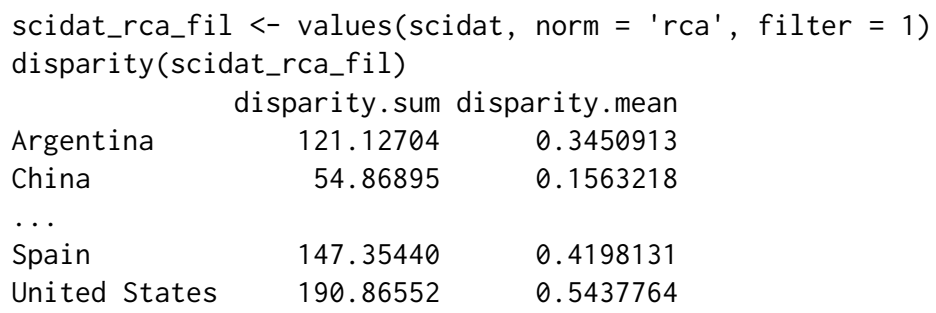

\section{Diversity measures that explicitly take variety, disparity and balance into account}

The package includes also "full" measures of diversity that are able to capture variety, balance and disparity at the same time. These measures are Rao and Rao-Stirling, where the former is widespread in ecology, while the latter is more commonly applied in social sciences and scientometrics (Rafols, 2014; Wang et al., 2015).

Both measures compute the sum of the multiplication of the distances (disparity) and the proportions (balance) between the pairs of two distinct categories $i$ and $j$ (see Table 1). However, Rao-Stirling diversity allows users to assign the weights/parameters $\alpha$ and $\beta$ according to the importance of the disparity or balance, respectively.

Rao diversity is equivalent to Rao-Stirling diversity with the parameter values $\alpha=\beta=1$. These values are also the default values in the function diversity(). Note that when the argument dis is not provided, the default method 'Euclidean distances' is used for the calculation of the dissimilarity matrix. Users can also provide their own dissimilarity matrix by using the argument dis in the function diversity().

In the following example from Scidat, we calculate Rao diversity as well as the Rao-Stirling diversity with the parameter values $\alpha=0.7$ and $\beta=0.3$ and cosine dissimilarities between the entities. This example shows that Rao-Stirling diversity provides the possibility to emphasize different aspects of diversity. When we use the Rao Index, then Spain is considered to be more "diverse" than the US, but when we assign more importance to disparity, by increasing the parameter $\alpha$ in the Rao-Stirling index, then the US is more "diverse."

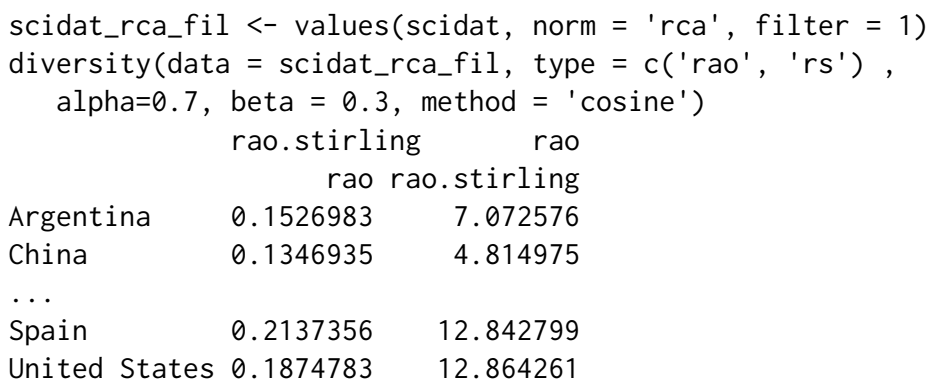

Thus, the Rao-Stirling index and the package diverse allows the user to analyze the impact of different similarity measures as well as different weights of disparity and balance on the resulting diversity values and rankings.

It must be noted that, so far, we focus in "diverse" on the Stirling taxonomy (Stirling, 2007, 1998). In ecology another set of "similarity-based" measures has been developed and can be accessed in the package entropart and treescape. 


\section{Synthetic data and performance tests}

In this Section, we show how to use diverse to create synthetic data to the level of individuals, entities and datasets. An individual is an independent object that belongs to a category (e.g. a paper in a certain discipline of Scidat or a person in a certain type of occupation in Pantheon). Entities are constituted by a set of categories and their values of abundance. A set of several entities constitutes a synthetic dataset for the type of diversity measures we apply in diverse.

The functions included in diverse to simulate data are sim_individuals(), sim_entities() and sim_dataset(). In each function the user can define the size (i.e. number of individuals), the required level of variety or richness and the method to define the distribution or values of abundance.

For example, if we want to create individuals of an entity, we can use the function sim_individuals() to generate synthetic data with 10000 individuals assigned to 50 different species (categories). Moreover, the values of absolute abundance of species can be, for instance, distributed according to a $\log$ normal distribution with $\mu=0.50$ and $\sigma=1.183$ (see histogram in Figure 5a and (Beck and Schwanghart, 2010)).

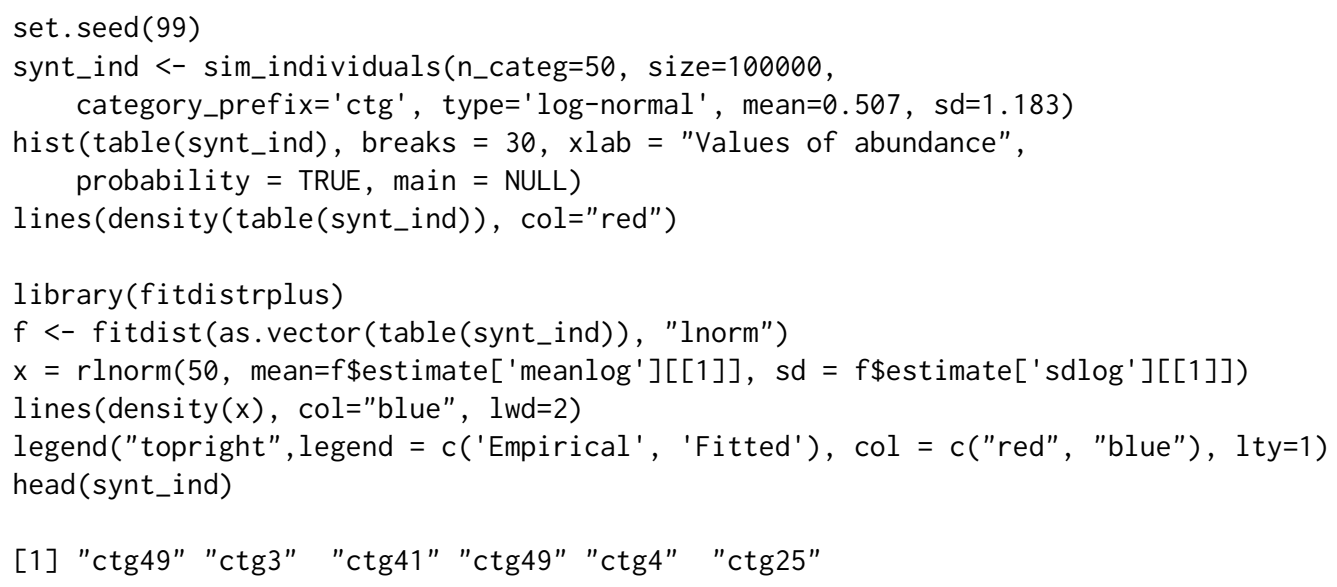

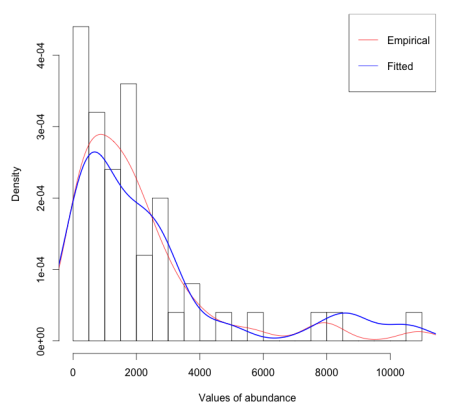

(a) Histogram of simulated $10 \mathrm{~K}$ individuals.

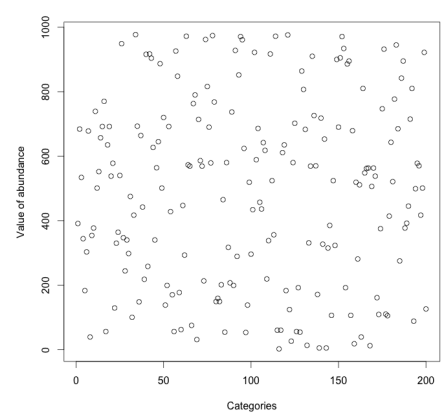

(b) Simulated entity with 200 categories.

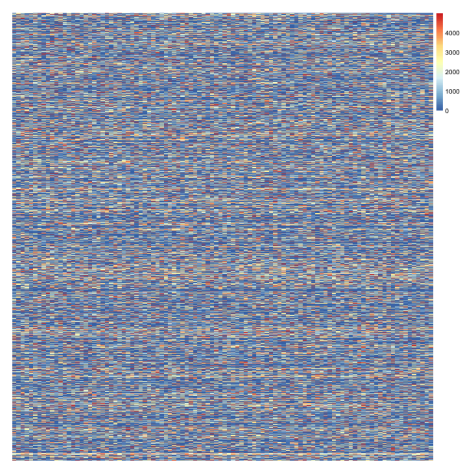

(c) Pheatmap of simulated dataset. $15 \mathrm{~K}$ entities, 100 categories. Colors according to value of abundance.

Figure 5: Analysis of simulated data.

If the user wants to generate a simulated entity with values of abundance produced by the aggregation of individuals in categories, diverse provides the function sim_entity (). This function allows the user to define a distribution and/or a required number of categories (n_categ()). See Figure $5 b$.

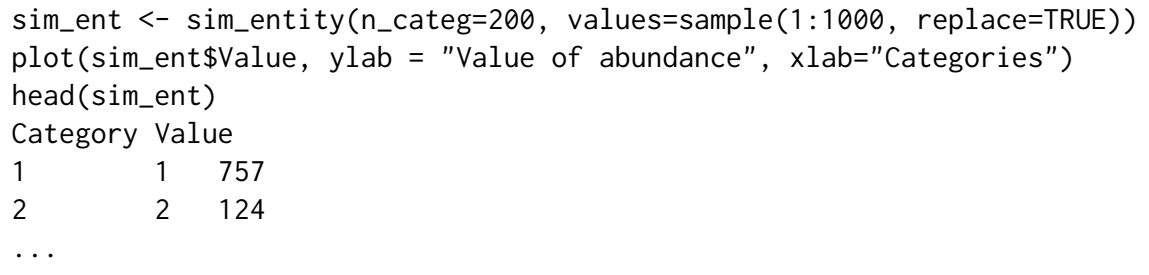


The simulation of a full dataset is also provided with diverse. The function sim_dataset() allows for users to define the number of categories in each entity (variety) as well as the number of required entities. A crucial argument of the function sim_dataset() is the vector of integers with the desired values of variety for each entity ( $n_{-}$categ). In the following example, we create a dataset of 1500 entities and 100 categories with random integer values of abundance between 10 and 5000 . The values of variety for each entity are also randomly sampled between 1 and 100. The resulting dataset is retrieved as a dataframe of values of abundance. By using the function values() we can plot this dataset as a matrix (see Figure 5c).

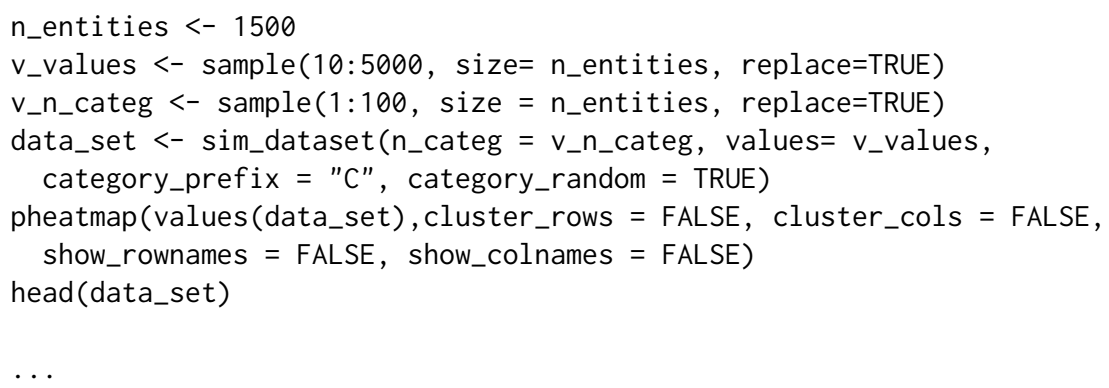

\section{Performance}

To test the performance of diverse we use the previously generated synthetic dataset (1500 entities by 100 categories), then we calculate the time used to perform two measures, namely Shannon entropy and Rao-Stirling diversity. Note that the second one is more time consuming since it involves the computation of a distance matrix. However, the time necessary to compute both measures is reasonable ( 0.021 and 2.697 seconds respectively). The time used to create the simulated dataset is more time consuming ( $\tilde{3} 5$ secs.) since the dataset must ensure that the assigned number of categories for each entity accomplishes the requirements. Still it is a reasonable amount of time considering the dimensions and characteristics of the obtained data.

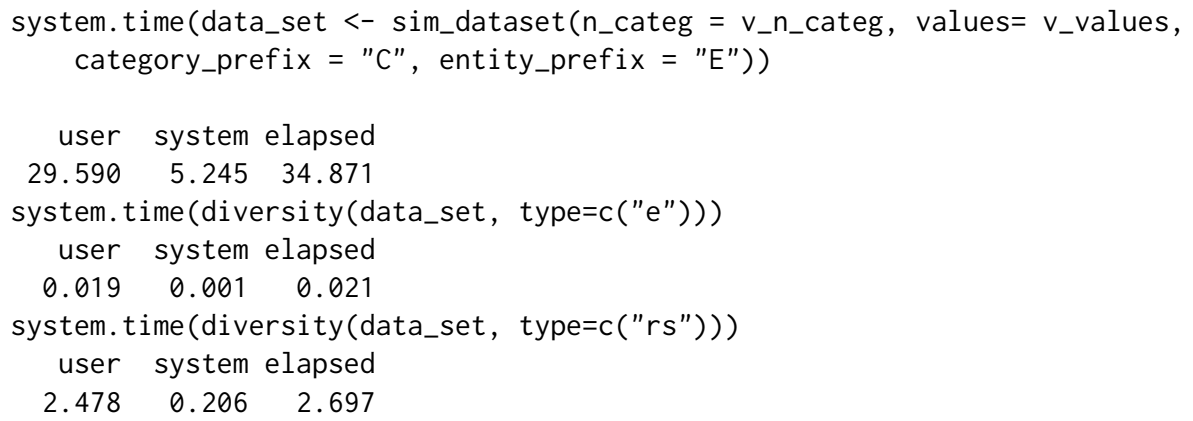

\section{Coverage, biases and caveats}

The package diverse is designed to work with datasets with a known number of categories and a comparatively low level of variety (i.e. scientific fields, occupations, or industrial sectors, in comparison to datasets in ecology with millions of species, including many unknown species). For instance, in ecology it has been demonstrated that diversity measures are biased in cases of small samples (e.g. in a very limited spatial area, limited amount of soil, etc.). Accordingly, in datasets on biodiversity, it is difficult to sample rare species appropriately (Beck and Schwanghart, 2010). To solve this issue associated to this type of datasets, measures of bias correction, e.g. of Shannon Entropy, have been proposed (Chao and Shen, 2003). These measures, mainly used in the area of ecology and biodiversity, are not yet implemented in diverse. Furthermore, considering for example phylogenetic diversity or functional diversity, other advanced measures, such as the generalization of the Rao's cuadratic entropy (Chao et al., 2014a), are not yet included. To address these current limitations it must be noted that diverse can also be used in combination with several specialized packages such as entropart, vegan or spadeR. For instance, diverse provides a function (to_entropart()) that allows the user to transform the datasets from the package diverse into values of abundance to be used in entropart. Here we present a simple example to compute the richness of the metacommunity (see the entropart manual for details (Marcon and Hérault, 2015)) generated with our synthetic dataset (the variable data_set of a previous example).

library (entropart)

abundance <- to_entropart(data_set) 
mc $<-$ MetaCommunity (abundance)

Richness (mc\$Ps); Shannon (mc\$Ps)

It must also be noted that the ability to statistically test differences in diversity measures across time and across systems could provide important insights for researchers. The following example shows a strong linear correlation between the Shannon Entropy in two different time steps of Scidat (dataframes scidat for 2013, and scidat_2 for 2003). Further statistical test functions need to be implemented in subsequent versions of of diverse, in order to also allow for the testing of the differences across systems. diverse will continue to learn from other disciplines, with the aim of implementing and adapting statistical test functions to the particular needs of researchers exploring the diversity in complex socioeconomic systems.

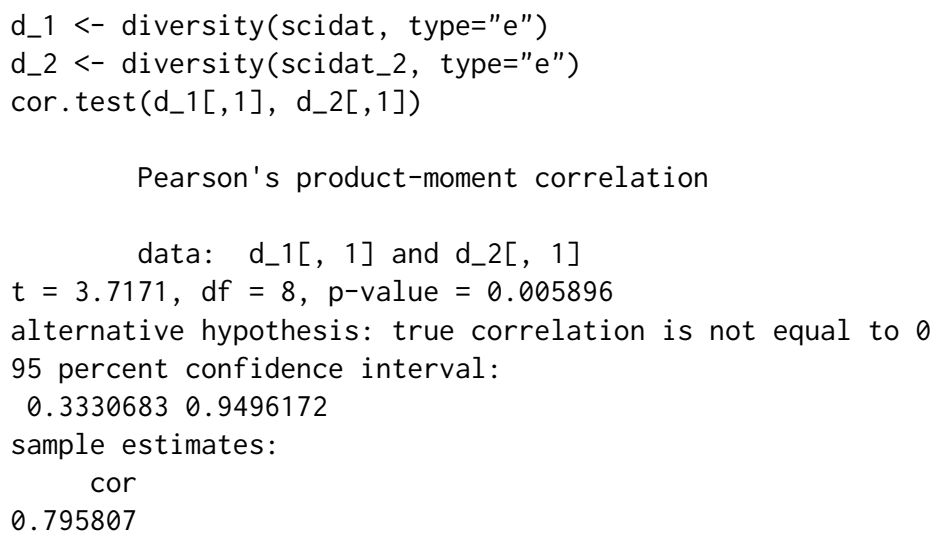

\section{Conclusions}

This paper introduced the package diverse which allows users to compute some of the most common measures of diversity from different fields of science. In summary, measuring diversity has become an important topic in many disciplines which analyze complex systems. The $\mathrm{R}$ package diverse allows for a combination of common measures from several disciplines and recent approaches from interdisciplinary research.

It must be noted that diverse has limitations that we aim to address in subsequent versions of the package. Possible future improvements include methods for considering diversity at different levels of aggregation (in hierarchical classification schemes, like mammals and insects, or agricultural or industrial goods, natural or social sciences, and their respective subcategories). Moreover, further emphasis on the role of different similarity measures at different levels of aggregations, as well as analyzing estimation error biases in incomplete samples are important future research areas in the measurement of diversity in socioeconomic systems, where social sciences can significantly learn from ecology and biology. Finally diverse can also continue to learn from ecology, biology, and other disciplines about how to apply statistical tests on the differences of diversity measures across systems.

In general, diverse offers a toolkit to analyze and visualize the diversity of entities, categories, and complex systems that is useful in particular for social scientists and interdisciplinary social research, as well as beginners in ecology and natural sciences. The package diverse provides different data import and export options and allows for the calculation of the different data transformations and similarity matrices, diversity measures, and diversity visualization options.

In order to present the functions provided by the package, we took advantage of an interdisciplinary taxonomy of diversity that defines variety, balance and disparity as three dimensions of diversity (Stirling, 2007). This taxonomy favors the creation of interdisciplinary bridges and helps in understanding how each diversity measure captures different aspects of diversity.

\section{Acknowledgments}

We would like to thank Ismael Rafols, Diego Chavarro, Daniele Rotolo, and Andy Stirling for the example codes and valuable comments on diversity measures. We are also grateful for the valuable comments made by two anonymous reviewers. MG and MM acknowledge the Program of Incentives to Scientific Initiation (PIIC) from DGIP at Universidad Técnica Federico Santa María. MG thanks internal project ING01-1516 from Universidad de Playa Ancha. DH acknowledges support from the Marie Curie International Outgoing Fellowship No. 328828 within the 7th European Community Framework Programme. MM and MG acknowledges support from project FONDECYT 11121435. 


\section{Bibliography}

B. Balassa. Trade Liberalisation and "Revealed" Comparative Advantage. The Manchester School, 33(2): 99-123, 1965. ISSN 1467-9957. doi: 10.1111/j.1467-9957.1965.tb00050.x. URL http: //onlinelibrary. wiley.com/doi/10.1111/j.1467-9957.1965.tb00050.x/abstract. [p65]

B. Balassa. Comparative advantage in manufactured goods: a reappraisal. The Review of Economics and Statistics, pages 315-319, 1986. [p65]

J. Beck and W. Schwanghart. Comparing measures of species diversity from incomplete inventories: an update. Methods in Ecology and Evolution, 1(1):38-44, Mar. 2010. ISSN 2041-210X. doi: 10.1111/j.2041-210X.2009.00003.x. URL http://onlinelibrary.wiley. com/doi/10.1111/j.2041210x.2009.00003.x/abstract. [p72,73]

W. H. Berger and F. L. Parker. Diversity of Planktonic Foraminifera in Deep-Sea Sediments. Science, 168(3937):1345-1347, Dec. 1970. ISSN 0036-8075, 1095-9203. doi: 10.1126/science.168.3937.1345. URL http: //www. sciencemag.org/content/168/3937/1345. [p66]

P. M. Blau. Inequality and heterogeneity: A primitive theory of social structure, volume 7. Free Press New York, 1977. [p66]

L. Ceriani and P. Verme. The origins of the Gini index: extracts from Variabilità e Mutabilità (1912) by Corrado Gini. The Journal of Economic Inequality, 10(3):421-443, June 2011. ISSN 1569-1721, 1573-8701. doi: 10.1007/s10888-011-9188-x. URL http://link. springer.com/article/10.1007/s10888-0119188-x. [p68]

A. Chao and T.-J. Shen. Nonparametric estimation of Shannon's index of diversity when there are unseen species in sample. Environmental and Ecological Statistics, 10(4):429-443, Dec. 2003. ISSN 1352-8505, 1573-3009. doi: 10.1023/ A:1026096204727. [p73]

A. Chao, C.-H. Chiu, and L. Jost. Unifying Species Diversity, Phylogenetic Diversity, Functional Diversity, and Related Similarity and Differentiation Measures Through Hill Numbers. Annual Review of Ecology, Evolution, and Systematics, 45(1):297-324, 2014a. doi: 10.1146/annurev-ecolsys120213-091540. URL http://dx.doi.org/10.1146/annurev-ecolsys-120213-091540. [p69,73]

A. Chao, N. J. Gotelli, T. Hsieh, E. L. Sander, K. Ma, R. K. Colwell, and A. M. Ellison. Rarefaction and extrapolation with Hill numbers: a framework for sampling and estimation in species diversity studies. Ecological Monographs, 84(1):45-67, 2014b. [p69]

D. Chavarro, P. Tang, and I. Rafols. Interdisciplinarity and research on local issues: evidence from a developing country. Research Evaluation, 23(3):195-209, 2014. [p60]

G. Csardi and T. Nepusz. The igraph software package for complex network research. InterJournal, Complex Systems:1695, 2006. URL http://igraph.org. [p62]

Z. Daróczy. Generalized information functions. Information and control, 16(1):36-51, 1970. [p69]

V. Debastiani and V. Pillar. SYNCSA - R tool for analysis of metacommunities based on functional traits and phylogeny of the community components. Bioinformatics, 28:2067-2068, 2012. [p60]

N. Eagle, M. Macy, and R. Claxton. Network Diversity and Economic Development. Science, 328 (5981):1029-1031, May 2010. ISSN 0036-8075, 1095-9203. doi: 10.1126/science.1186605. URL http://science.sciencemag.org/content/328/5981/1029. [p60]

J. Farchy and H. Ranaivoson. Measuring the Diversity of Cultural Expressions: Applying the Stirling Model of Diversity in Culture. Technical Report 6, Unesco Institute for Statistics, 2011. [p60]

K. Frenken, F. V. Oort, and T. Verburg. Related variety, unrelated variety and regional economic growth. Regional Studies, 41(5):685-697, July 2007. ISSN 0034-3404. doi: 10.1080/00343400601120296. URL http://dx.doi .org/10.1080/00343400601120296. [p60]

C. Gini. Variabilità e Mutuabilità. Contributo allo Studio delle Distribuzioni e delle Relazioni Statistiche. Bologna, c. cuppini edition, 1912. [p66]

M. R. Guevara, D. Hartmann, M. Aristarán, M. Mendoza, and C. A. Hidalgo. The research space: using career paths to predict the evolution of the research output of individuals, institutions, and nations. Scientometrics, pages 1-15, Sept. 2016. ISSN 0138-9130, 1588-2861. doi: 10.1007/s11192-016-2125-9. URL http://link. springer. com/article/10.1007/s11192-016-2125-9. [p60,70] 
D. Hartmann, M. R. Guevara, C. Jara-Figueroa, M. Aristarán, and C. A. Hidalgo. Linking Economic Complexity, Institutions and Income Inequality. arXiv:1505.07907 [physics, q-fin], 2016. URL http://arxiv.org/abs/1505.07907. arXiv: 1505.07907. [p70]

D. M. A. Haughton and S. Mukerjee. The economic measurement and determinants of diversity. Social Indicators Research, 36(3):201-225, Nov. 1995. ISSN 0303-8300, 1573-0921. doi: 10.1007/BF01078814. URL http://link. springer.com/article/10.1007/BF01078814. [p60]

R. Hausmann, C. A. Hidalgo, S. Bustos, M. Coscia, S. C. (M.A.), J. Jimenez, A. Simões, and M. A. Yıldırım. The Atlas of Economic Complexity: Mapping Paths to Prosperity. Center for International Development, Harvard University, 2011. ISBN 978-0-615-54662-9. [p62]

J. Havrda and F. Charvát. Quantification method of classification processes. concept of structural a-entropy. Kybernetika, 03(1):(30)-35, 1967. URL http://eudml .org/doc/28681. [p66, 69]

C. Hidalgo. Why Information Grows: The Evolution of Order, from Atoms to Economies. Basic Books, June 2015. ISBN 978-0-465-04899-1. [p69]

C. A. Hidalgo and R. Hausmann. The building blocks of economic complexity. Proceedings of the National Academy of Sciences, 106(26):10570-10575, June 2009. ISSN 0027-8424, 1091-6490. doi: 10.1073/pnas.0900943106. URL http://www. pnas.org/content/106/26/10570. [p60]

C. A. Hidalgo, B. Klinger, A.-L. Barabási, and R. Hausmann. The product space conditions the development of nations. Science, 317(5837):482-487, July 2007. ISSN 0036-8075, 1095-9203. doi: 10.1126/science.1144581. URL http://www. sciencemag. org/content/317/5837/482. [p60, 62, 67, $70]$

M. O. Hill. Diversity and Evenness: A Unifying Notation and Its Consequences. Ecology, 54(2):427-432, Mar. 1973. ISSN 0012-9658. doi: 10.2307/1934352. URL http://www. esajournals. org/doi/abs/10. 2307/1934352. [p66, 69]

C. J. C. J. Humphries, P. L. Forey, R. I. Vane-Wright, and S. Association. Systematics and conservation evaluation, 1994. URL http://bvbr.bib-bvb.de:8991/F?func=service\&doc_library=BVB01\& local_base=BVB01\&doc_number=006426531\&line_number=0001\&func_code=DB_RECORDS\&service _ type=MEDIA. Published for the Systematics Association. [p60]

T. Jombart, M. Kendall, J. Almagro-Garcia, and C. Colijn. treescape: Statistical Exploration of Landscapes of Phylogenetic Trees. 2016. URL http://CRAN. R-project.org/package=treescape. R package version 1.8.16. [p60]

L. Jost. Entropy and diversity. Oikos, 113(2):363-375, May 2006. ISSN 1600-0706. doi: 10.1111/j. 2006.0030-1299.14714.x. URL http://onlinelibrary.wiley.com/doi/10.1111/j.2006.0030-1299. 14714.x/abstract. [p69]

L. Jost. The Relation between Evenness and Diversity. Diversity, 2(2):207-232, Feb. 2010. doi: 10.3390/d2020207. URL http://www. mdpi.com/1424-2818/2/2/207. [p68]

K. Keenan, P. McGinnity, T. F. Cross, W. W. Crozier, and P. A. Prodöhl. diveRsity: An R package for the estimation of population genetics parameters and their associated errors. Methods in Ecology and Evolution, 4(8):782-788, 2013. doi: 10.1111/2041-210X.12067. URL http: //onlinelibrary.wiley. com/doi/10.1111/2041-210X.12067/abstract. R package version 1.9.89. [p60]

R. Kindt and R. Coe. Tree diversity analysis. A manual and software for common statistical methods for ecological and biodiversity studies. World Agroforestry Centre (ICRAF), Nairobi (Kenya), 2005. URL http://www. worldagroforestry.org/treesandmarkets/tree_diversity_analysis.asp. ISBN 929059-179-X. [p60, 61]

R. Kolde. pheatmap: Pretty Heatmaps. 2015. URL http://CRAN. R-project. org/package=pheatmap. R package version 1.0.7. [p61]

L. I. Kuncheva and C. J. Whitaker. Measures of Diversity in Classifier Ensembles and Their Relationship with the Ensemble Accuracy. Machine Learning, 51(2):181-207, May 2003. ISSN 0885-6125, 1573-0565. doi: 10.1023/A:1022859003006. [p60]

E. Laliberté and P. Legendre. A distance-based framework for measuring functional diversity from multiple traits. Ecology, 91(1):299-305, 2010. ISSN 0012-9658. doi: 10.1890/08-2244.1. URL http://www. esajournals.org. ezproxy. canterbury.ac.nz/doi/abs/10.1890/08-2244.1. [p60]

Macro Connections MIT MediaLab. Pantheon - Mapping historical cultural production, 2014. URL http://pantheon.media.mit.edu/methods. [p61] 
E. Marcon and B. Hérault. entropart: An R Package to Measure and Partition Diversity. Journal of Statistical Software, 67(8):1-26, 2015. doi: 10.18637/jss.v067.i08. [p60, 61,73]

Z. Marion, J. Fordyce, and B. Fitzpatrick. hierDiversity: Hierarchical Multiplicative Partitioning of Complex Phenotypes. 2015. URL http://CRAN.R-project.org/package=hierDiversity. R package version 0.1. [p60]

D. G. Mcdonald and J. Dimmick. The Conceptualization and Measurement of Diversity. Communication Research, 30(1):60-79, Jan. 2003. ISSN 0093-6502, 1552-3810. doi: 10.1177/0093650202239026. URL http://crx. sagepub.com/content/30/1/60. [p62]

D. Meyer and C. Buchta. proxy: Distance and Similarity Measures. 2015. URL http://CRAN. R-project. org/package=proxy. R package version 0.4-15. [p61]

Nature. Diversity challenge. Nature, 513(7518):279-279, Sept. 2014. ISSN 0028-0836, 1476-4687. doi: 10.1038/513279a. URL http://www. nature.com/doifinder/10.1038/513279a. [p60]

S. V. Nederland. Sovon Home Page, 2015. URL https://www. sovon. nl/en. [p61]

J. Oksanen, F. G. Blanchet, R. Kindt, P. Legendre, P. R. Minchin, R. B. O'Hara, G. L. Simpson, P. Solymos, M. H. H. Stevens, and H. Wagner. vegan: Community Ecology Package. 2016. URL http://CRAN. Rproject. org/package=vegan. R package version 2.3-5. [p60]

E. C. Pielou. Introduction to Mathematical Ecology. John Wiley \& Sons Inc, New York, Jan. 1970. ISBN 978-0-471-68918-8. [p66, 68]

M. Pietrzak, M. Seweryn, and G. Rempala. divo: Tools for Analysis of Diversity and Similarity in Biological Systems. 2016. URL http://CRAN. R-project. org/package=divo. R package version 0.1.2. [p60]

R Core Team. foreign: Read Data Stored by Minitab, S, SAS, SPSS, Stata, Systat, Weka, dBase, ... 2015. URL http: //CRAN.R-project.org/package=foreign. R package version 0.8-65. [p64]

I. Rafols. Knowledge Integration and Diffusion: Measures and Mapping of Diversity and Coherence. In Y. Ding, R. Rousseau, and D. Wolfram, editors, Measuring Scholarly Impact, pages 169-190. Springer International Publishing, 2014. ISBN 978-3-319-10376-1 978-3-319-10377-8. URL http: //link. springer. com/chapter/10.1007/978-3-319-10377-8_8. [p60,71]

I. Rafols and M. Meyer. Diversity and network coherence as indicators of interdisciplinarity: case studies in bionanoscience. Scientometrics, 82(2):263-287, June 2009. ISSN 0138-9130, 1588-2861. doi: 10.1007/s11192-009-0041-y. URL http://link. springer. com/article/10.1007/s11192-009$0041-y \cdot[\mathrm{p} 70]$

I. Rafols, A. L. Porter, and L. Leydesdorff. Science overlay maps: A new tool for research policy and library management. Journal of the American Society for Information Science $\mathcal{E}$ Technology, 61(9):1871-1887, Sept. 2010. ISSN 15322882. doi: 10.1002/asi.21368. URL http://libproxy.mit.edu/login?url=http://search. ebscohost.com/login.aspx?direct= true \&db=bth\&AN=53286068\&si te=eds-live. $[p 60,62,63,70]$

C. R. Rao. Diversity and dissimilarity coefficients: A unified approach. Theoretical Population Biology, 21(1):24-43, Feb. 1982. ISSN 0040-5809. doi: 10.1016/0040-5809(82)90004-1. URL http://www. sciencedirect.com/science/article/pii/0040580982900041. [p62,66]

S. A. Rhoades. Herfindahl-Hirschman Index, The. Federal Reserve Bulletin, 79:188, 1993. URL http: //heinonline.org/HOL/Page?handle=hein. journals/fedred79\&id=376\&div=\&collection=. [p66]

A. Rényi. On Measures of Entropy and Information. The Regents of the University of California, 1961. URL http://projecteuclid.org/euclid.bsmsp/1200512181. [p66]

R. Scherer and P. Pallmann. simboot: Simultaneous inference for diversity indices. 2014. URL http: //CRAN. R-project. org/package=simboot. R package version 0.2-5. [p60]

SCImago. Scimago Journal \& Country Rank, 2007. URL http://www. scimagojr.com/. [p61]

C. E. Shannon. A mathematical theory of communication. The Bell System technical Journal, 27: 379-423,623-656, Oct. 1948. [p66, 69]

E. H. Simpson. Measurement of diversity. Nature, 163, 1949. [p66]

A. Stirling. On the economics and analysis of diversity. Science Policy Research Unit (SPRU), Electronic Working Papers Series, Paper, 28:1-156, 1998. [p62,71] 
A. Stirling. A general framework for analysing diversity in science, technology and society. Interface The Journal of Royal Society, 4(15):707-719, Aug. 2007. [p61, 62, 66, 70, 71, 74]

M. Tennekes. treemap: Treemap Visualization. 2016. URL http://CRAN.R-project.org/package= treemap. R package version 2.4-1. [p62]

C. Tsallis. Possible generalization of Boltzmann-Gibbs statistics. Journal of Statistical Physics, 52(1-2): 479-487, 1988. [p66, 69]

H. Tuomisto. An updated consumer's guide to evenness and related indices. Oikos, 121(8):1203-1218, Aug. 2012. ISSN 1600-0706. doi: 10.1111/j.1600-0706.2011.19897.x. URL http://onlinelibrary . wiley.com/doi/10.1111/j.1600-0706.2011.19897.x/abstract. [p68]

C. S. Wagner, J. D. Roessner, K. Bobb, J. T. Klein, K. W. Boyack, J. Keyton, I. Rafols, and K. Börner. Approaches to understanding and measuring interdisciplinary scientific research (IDR): A review of the literature. Journal of Informetrics, 5(1):14-26, Jan. 2011. ISSN 1751-1577. doi: 10.1016/j.joi.2010. 06.004. URL http://www. sciencedirect.com/science/article/pii/S1751157710000581. [p60]

C. Wang, M. Genkin, G. Berry, L. Chen, and M. Brashearswork. Blaunet: Calculate and Analyze Blau Status for Measuring Social Distance. 2016. URL http://CRAN. R-project. org/package=Blaunet. R package version 2.0.4. [p60]

J. Wang, B. Thijs, and W. Glänzel. Interdisciplinarity and Impact: Distinct Effects of Variety, Balance, and Disparity. PLoS ONE, 10(5):e0127298, May 2015. doi: 10.1371/journal.pone.0127298. URL http://dx.doi.org/10.1371/journal. pone.0127298. [p71]

A. Z. Yu, S. Ronen, K. Hu, T. Lu, and C. A. Hidalgo. Pantheon 1.0, a manually verified dataset of globally famous biographies. Scientific data, 3, 2016. [p61]

Miguel R. Guevara

Computer Science Department, Universidad de Playa Ancha, and

Department of Informatics, Universidad Técnica Federico Santa María

Valparaíso

Chile

miguel.guevara@upla.cl

Dominik Hartmann

Chair of Innovation Management and Innovation Economics, University of Leipzig

Grimmaische Straße 12, 04109, Leipzig

Fraunhofer Center for International Management and Knowledge Economy

Neumarkt 9-19, 04109, Leipzig

Germany

dominik. hartmann@uni-leipzig.de

Marcelo Mendoza

Department of Informatics, Universidad Técnica Federico Santa María

Av. Vicuna Mackeña 3939, San Joaquín, Santiago

Chile

mmendoza@inf.utfsm.cl 